\title{
Detailed Illustration of a Patient With Ipsilateral Seizures
}

\author{
Halil Ondera, c, F. Irsel Tezer ${ }^{b}$
}

\section{To the Editor}

A 37-year-old male patient with a history of surgical resection of diffuse astrocytoma right frontoparietal lobe was admitted to our policlinic due to complex partial seizures characterized by focal convulsions on the right hand. Seizures were in the form of convulsions of right hand following a sensation-like ringing in the left ear and were generally lasting for a few minutes resulting in postictal confusion. His seizures had started a few months prior to the diagnosis of astrocytoma 4 months ago. Successful resective surgery was performed and postoperative radiotherapy (1 month after the diagnosis of tumor) was administered. However, he had been having seizures nearly twice a week despite taking the anti-epileptic medication of topiramate $300 \mathrm{mg}$ daily. On neurological examination, he was fully orientated and cooperated. Motor, sensory and cerebellar examinations were within normal limits. Deep tendon reflexes were evaluated as moderately brisk on the left side. Routine electroencephalogram (EEG) showed right centro-parietal paroxysmal disturbances characterized by sharp and slow waves (Fig. 1). Imaging (Fig. 2a, b) and electrophysiological evidence remarked an underlying right hemisphere focus; however, in contrary to this, he suffered from motor seizures of right hand constantly. At this point, to evaluate a possible variation in corticospinal tracts, diffusion tensor imaging (DTI) was performed which demonstrated bilateral corticospinal tracts with normal crossing at the decussation (Fig. 2c). Carbamazepine $600 \mathrm{mg}$ daily was added to the treatment regimen which provided a significant improvement in seizures and the patient was evaluated as seizure-free on the third month of follow-up.

Ipsilateral seizures have been reported very rarely in the literature $[1,2]$. In these reports, pathophysiological explanations such as possible anatomical variations in the crossing of pyramidal fibers, one dominant cerebral hemisphere taking over the function of both sides of the body and colosally mediated inhibitory system over the uncrossed pyramidal fibers have been suggested [1]. In our patient, EEG showed epileptiform discharges in the lesional hemisphere. On the other hand, DTI showed normal crossing of bilateral corticospinal tract at

Manuscript submitted March 22, 2019, accepted April 26, 2019

aDepartment of Neurology, Yozgat City Hospital, Yozgat, Turkey b Department of Neurology, Hacettepe University Hospital, Ankara, Turkey ${ }^{\mathrm{c} C}$ Corresponding Author: Halil Onder, Department of Neurology, Yozgat City Hospital, Yozgat, Turkey. Email: halilnder@yahoo.com

doi: https://doi.org/10.14740/jnr526 the decussation which suggested mechanisms other than variations in the crossing of pyramidal fibers.

In the interesting report by Kim et al, the ipsilateral motor pathway from the unaffected motor cortex to the affected hand was demonstrated by transcranial magnetic stimulation (TMS) method [3]. They remarked the ipsilateral motor pathway as an important mechanism in terms of stroke rehabilitation. They also discussed the possible role of the ipsilateral corticospinal tract and non-corticospinal tract in this finding of ipsilateral motor responses determined in the TMS studies. However, the role of ipsilateral motor activity in the control of upper extremity movements remains controversial [4]. Although there are evidences supporting function of the activation of the ipsilateral motor cortex in motor output of unilateral motor tasks, mechanisms by which the motor cortex innervates the ipsilateral spinal motor neurons and clinical significance of this pathway remain unclear $[4,5]$. Forefront hypotheses to explain ipsilateral motor cortex function were: first, via transcallosal interneurons that transmit from the primarily active motor cortex to the contralateral motor cortex; second, possible activation of both hemisphere motor cortex induced by initiation of an action, and subsequent inhibition of the ipsilateral motor cortex by opposite side motor cortex by means of interhemispheric pathways $[4,6]$. On the other hand, in literature, left hemisphere has been shown to play a greater role in ipsilateral motor control than the right hemisphere, which was not the case in our report (right hemisphere ipsilateral cerebral event is presented) [7]. Of note, these conclusions are derived from studies conducted on stroke patients and normal individuals, which completely differ from epilepsy in terms of pathophysiology. Hence, at some point, it may not be rational to draw conclusions based on these studies. Nonetheless, reports of studies on epilepsy patients using advanced paraclinical methods still lack in the literature.

A crucial discussion may be that possible extension of epileptiform discharges towards contralateral hemisphere might be leading to the ipsilateral tonic-clonic seizures. In this setting, a valid hypothesis of why contralateral seizures had not occurred in our patient may be explained through disturbed corticospinal pathways preventing transformation of ictal activity in the form of clinical contralateral convulsive seizures. Nonetheless, electrophysiological investigations (motorevoked potentials and video EEG monitorization) could not be performed because the patient had not given consent. Hence, these explanations can only be hypothesized. In conclusion, here, we illustrate a strictly interesting patient manifesting with ipsilateral convulsive seizures by detailed clinical as well as DTI and routine EEG findings. Future studies of larger case series using combine para-clinic methods are needed to clarify these arguments. These reports may also provide substantial 


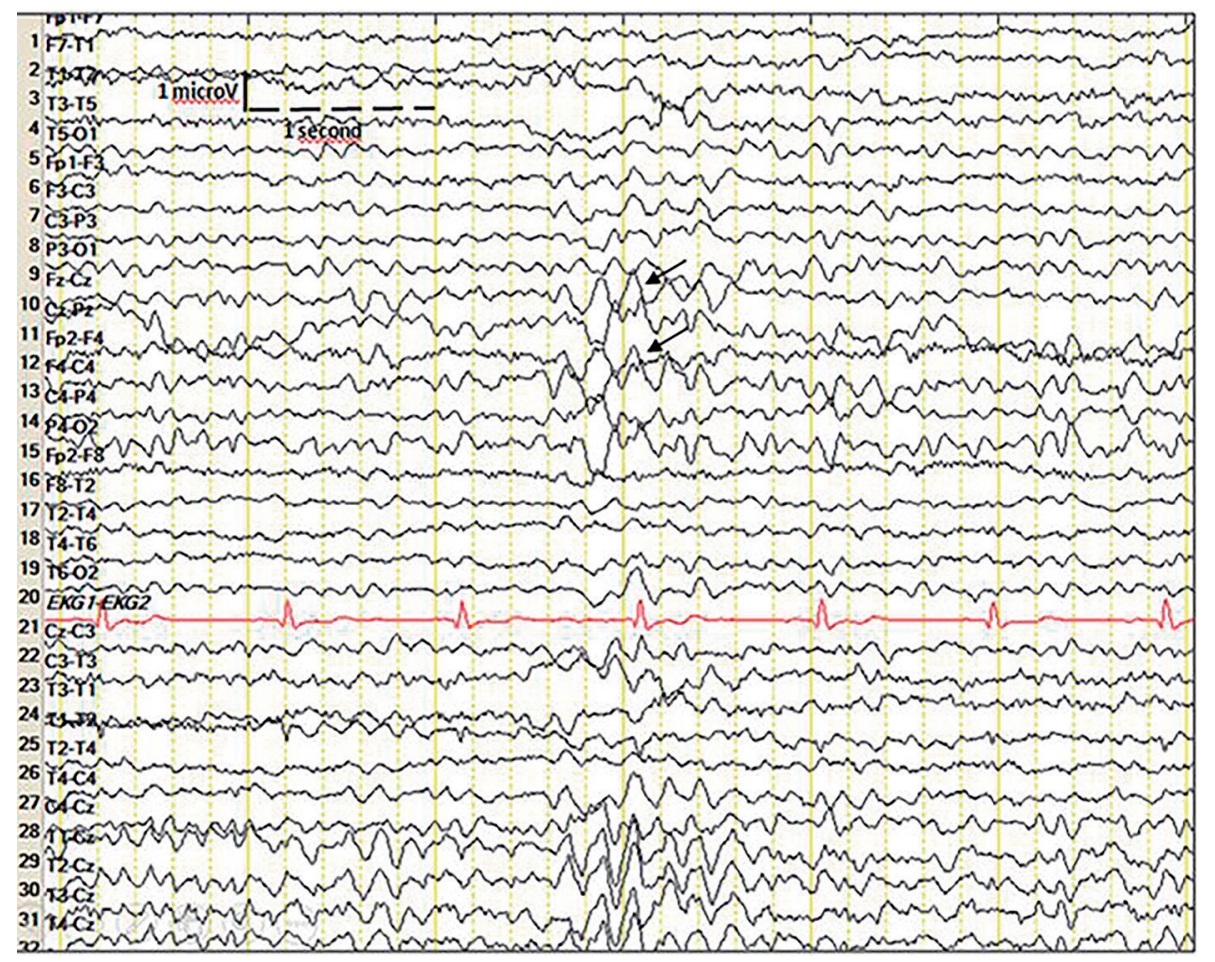

Figure 1. Routine EEG shows right centro-parietal paroxysmal disturbances characterized by sharp and slow waves. EEG: electroencephalogram.

contributions to our understanding of the physiology of corticospinal pathways in normal and pathological backgrounds.

\section{Acknowledgments}

None to declare.

\section{Financial Disclosure}

None to declare.

\section{Conflict of Interest}

The authors declare no conflict of interest.

\section{Informed Consent}

Not applicable.

\section{Author Contributions}

HO: design of the study, reporting of the routine EEG recordings, data analysis and writing of manuscript. IT: design of the study, analyses of the ECG recordings during routine EEG, reporting of the routine EEG recordings and writing of manuscript.

\section{References}

1. Lekhra OP, Bansal SK, Prabhakar S, Chopra JS. Ipsilateral cerebral events. Neurol India. 1995;43(2):96-100.

2. Kofman O, Tasker R. Ipsilateral and focal inhibitory seizures. Neurology. 1967;17(11):1082-1086.

3. Kim YH, Jang SH, Byun WM, Han BS, Lee KH, Ahn SH. Ipsilateral motor pathway confirmed by combined brain mapping of a patient with hemiparetic stroke: a case report. Arch Phys Med Rehabil. 2004;85(8):1351-1353.

4. Ghacibeh GA, Mirpuri R, Drago V, Jeong Y, Heilman KM, Triggs WJ. Ipsilateral motor activation during unimanual and bimanual motor tasks. Clin Neurophysiol. 2007;118(2):325-332.

5. Muellbacher W, Facchini S, Boroojerdi B, Hallett M. Changes in motor cortex excitability during ipsilateral hand muscle activation in humans. Clin Neurophysiol. 2000;111(2):344-349.

6. Ziemann U, Ishii K, Borgheresi A, Yaseen Z, Battaglia F, Hallett M, Cincotta M, et al. Dissociation of the pathways mediating ipsilateral and contralateral motor-evoked potentials in human hand and arm muscles. J Physiol. 1999;518(Pt 3):895-906.

7. Ziemann U, Hallett M. Hemispheric asymmetry of ipsilateral motor cortex activation during unimanual motor tasks: further evidence for motor dominance. Clin Neurophysiol. 2001;112(1):107-113. 

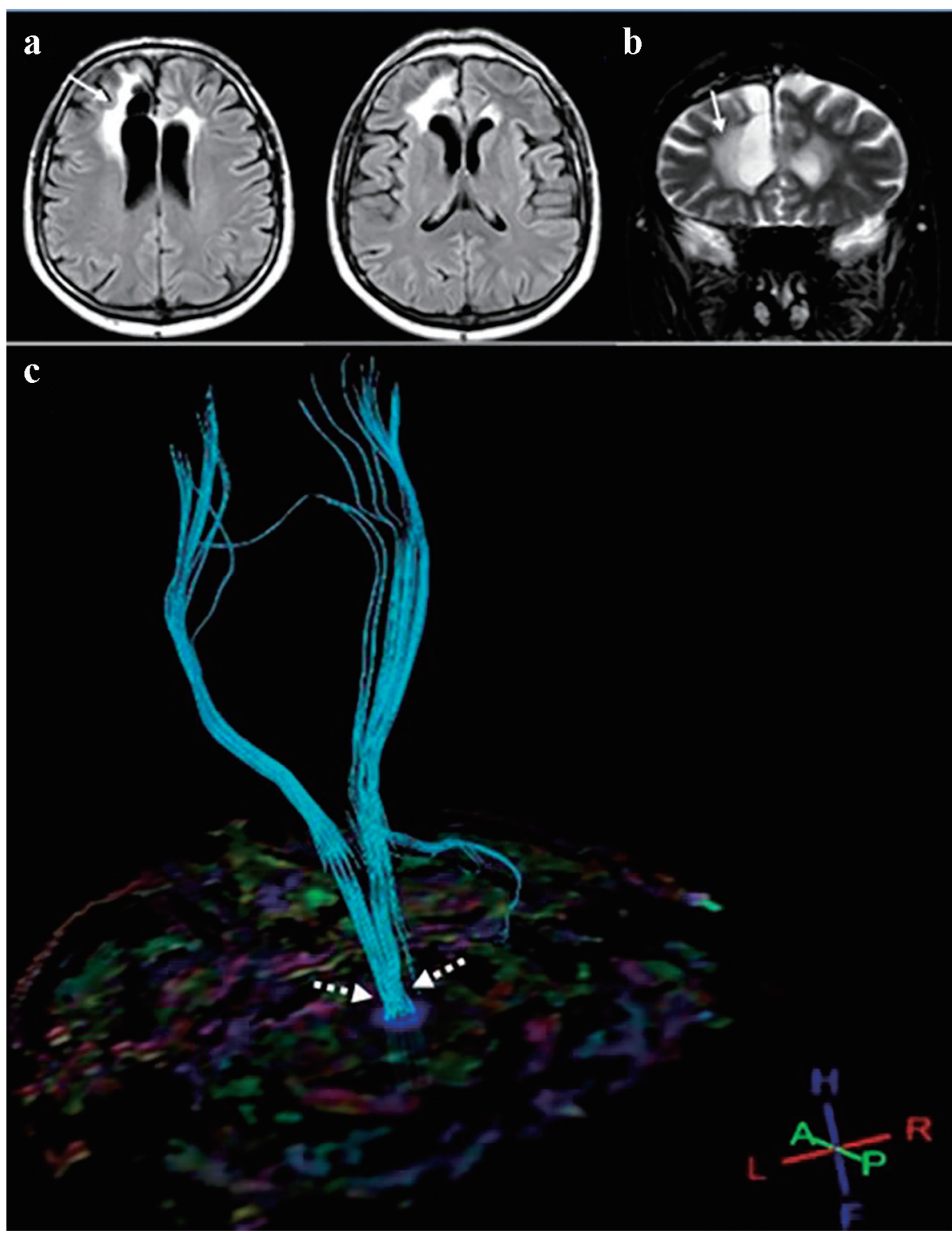

Figure 2. Cranial MRI shows encephalomalacia in the right frontal lobe (axial FLAIR images (a) and coronal T2-weighted image (b)). Diffusion tensor imaging of the brain, showing bilateral corticospinal tracts with normal crossing at the decussation (c). MRI: magnetic resonance imaging; FLAIR: fluid-attenuated inversion recovery. 